Original Research

\title{
Effects of a national policy advocating rational drug use on decreases in outpatient antibiotic prescribing rates in Thailand
}

\author{
Onanong WALEEKHACHONLOET ${ }^{(D)}$, Thananan RATTANACHOTPHANIT(D), Chulaporn LIMWATTANANON (D), \\ Noppakun THAMMATACHAREE (iD), Supon LIMWATTANANON (iD). \\ Received (first version): 23-Oct-2020 Accepted: 24-Jan-2021 Published online: 9-Feb-2021
}

\begin{abstract}
Objective: This study examined the effects of a national policy advocating rational drug use (RDU), namely, the 'RDU Service Plan', starting in fiscal year 2017 and implemented by the Thai Ministry of Public Health (MOPH), on trends in antibiotic prescribing rates for outpatients. The policy was implemented subsequent to a voluntary campaign involving 136 hospitals, namely, the 'RDU Hospital Project', which was implemented during fiscal years 2014-2016.

Methods: Hospital-level antibiotic prescribing rates in fiscal years 2014-2019 for respiratory infections, acute diarrhea, and fresh wounds were aggregated for two hospital groups using equally weighted averages: early adopters of RDU activities through the RDU Hospital Project and late adopters under the RDU Service Plan. Pre-/post-policy annual changes in the prescribing levels and trends were compared between the two groups using an interrupted time-series analysis.

Results: In fiscal years 2014-2016, decreases in antibiotic prescribing rates for respiratory infections and acute diarrhea in both groups reflected a trend that existed before the RDU Service Plan was implemented. The immediate effect of the RDU Service Plan policy occurred in fiscal year 2017, when the prescribing level among the late adopters dropped abruptly for all three conditions with a greater magnitude than in the decrease among the early adopters, despite nonsignificant differences. The medium-term effect of the RDU Service Plan was identified through a further decreasing trend during fiscal years 2017-2019 for all conditions in both groups, except for acute diarrhea among the early adopters.

Conclusions: The national policy on rational drug use effectively reduced antibiotic prescribing for common but questionable
\end{abstract} outpatient conditions.

Keywords

Anti-Bacterial Agents; Outpatients; Hospitals; Inappropriate Prescribing; Drug Resistance, Bacterial; Public Health; Policy; Diffusion of Innovation; Change Management; Interrupted Time Series Analysis; Thailand

\section{INTRODUCTION}

In Thailand, antibiotic consumption per 1,000 inhabitants per day was 18.0 defined daily doses (DDDs), close to the median (19.5) DDDs reported for 76 countries in 2015 . $^{1}$ Economically, expenditure on antibiotics accounted for approximately one-tenth of total medicine expenditure, which is much higher than that in developed countries, for example, $2 \%$ in the US. ${ }^{2-4}$ Half $(50.3 \%)$ of household members who used antibiotics reportedly obtained the drugs from public hospitals and health facilities. ${ }^{5}$ Antibiotic prescribing rates, however, tend to vary widely across hospitals. ${ }^{2}$ Due to concerns regarding antibiotic overuse, an action research program aiming to change the perceptions and prescribing behavior of physicians, known as the "Antibiotic Smart Use (ASU)" program, was trialed in 2007 2012. The ASU program focused on three questionable

\footnotetext{
Onanong WALEEKHACHONLOET. PhD. Faculty of Pharmacy, Mahasarakham University. Maha Sarakham (Thailand). ornanong.w@msu.ac.th

Thananan RATTANACHOTPHANIT. PhD. Faculty of Pharmacy, Mahasarakham University. Maha Sarakham (Thailand). tananan.r@msu.ac.th

Chulaporn LIMWATTANANON. PhD. Faculty of Pharmaceutical Sciences, Khon Kaen University. Khon Kaen (Thailand). limw0002@kku.ac.th

Noppakun THAMMATACHAREE. PhD. Health Systems Research Institute. Bangkok (Thailand).noppakun@hsri.or.th

Supon LIMWATTANANON. PhD. Faculty of Pharmaceutical

Sciences, Khon Kaen University. Khon Kaen (Thailand).

supon@kku.ac.th
}

conditions common to outpatient visits: respiratory infections (RIs) that involve the upper respiratory tract and acute bronchitis, acute diarrhea and fresh wounds. ${ }^{6-7}$ In addition to research activities in hospitals, the ASU program included a widespread campaign for public awareness.

In the beginning of fiscal year 2014 (October 2013), the ASU program led by pharmacist team leaders in the Thai Food and Drug Administration (FDA) was scaled up from an action research program to a hospital-wide campaign called the "Rational Drug Use (RDU) Hospital Project". The project involved collaborations with public organizations, professional associations, and academia. The main strategy included a campaign for raising awareness and concerns among health professionals and patients through hospital networking; in this regard, the RDU handbook was a major tool. ${ }^{8-10}$ The key RDU activities led by the hospitals' drug and therapeutic committees included developing hospital strategies to encourage prescribing behaviors that conformed to the RDU criteria, monitoring and providing feedback regarding prescribing information to individual prescribers, and strengthening the capacity of hospital staff for data analysis. The lessons learned concerning the progress made and obstacles encountered in the RDU Hospital Project were shared throughout the hospital network on a regular basis. One hundred thirty-six hospitals affiliated with the Ministry of Public Health (MOPH) joined the RDU Hospital Project and adopted RDU activities on a voluntary basis. 
Aiming for improved quality in the use of medicines, the features of the RDU Hospital Project were adopted by the MOPH and fully integrated into the Ministry's service plan policies at the beginning of fiscal year 2017 (October 2016). The project was then renamed the "RDU Service Plan", and its implementation as a ministerial policy was expanded to the remaining $\mathrm{MOPH}$ hospitals nationwide that did not participate in the RDU Hospital Project. Hospital directors adopted the RDU Service Plan policies through their leading roles in establishing hospital strategies to achieve the RDU goals, encouraging hospital staff to conduct clinical practice according to the policy recommendations, and developing an internal monitoring and evaluation system. The RDU Service Plan prioritized antibiotic prescribing for three outpatient conditions, including RIs, acute diarrhea, and fresh wounds. ${ }^{11}$ The use of antibiotics for RIs and acute diarrhea is among the WHO indicators for the quality use of medicine. $^{12}$ For RIs, the European Surveillance of Antimicrobial Consumption project developed diseasespecific quality indicators for antibiotic prescribing for outpatients and set the target to be no greater than $20 \%{ }^{13}$ Antibiotic use for prophylaxis in fresh wounds is usually reserved for cases with a high risk of infection. ${ }^{14-15}$ In practice, however, the use of antibiotics for fresh wounds is common in outpatient care settings where physicians are not aware of the recommendations and might suspect a bacterial infection that may lead to serious complications. The RDU Service Plan aimed for antibiotic prescribing rates for no more than $20 \%$ of diagnosed $\mathrm{RI}$ and acute diarrhea cases and $40 \%$ of fresh wound cases. ${ }^{11}$

To monitor hospital service quality through key performance indicators, all MOPH district and provincial hospitals were persuaded to submit data on service outputs, including inpatient admissions, outpatient visits, and drug prescriptions, to the MOPH Health Data Center (HDC) beginning in October $2013 .^{16}$ The prescribing rates for antibiotics in outpatient visits for the target conditions were calculated over a fiscal year $\left(1^{\text {st }}\right.$ October of previous calendar year until $30^{\text {th }}$ September of current calendar year) for every hospital and were used by the MOPH inspectors general to supervise hospital administrators on compliance with the ministerial service plan policies.

Making changes in prescribing behavior require acknowledging evidence-based clinical practice guidelines and reinforcing the positive motivation of individual practitioners. Both the RDU Hospital Project and the RDU Service Plan were policy agendas in which clinical and health system pharmacists were involved in advocating the rational use of antibiotics for common conditions. The present study aimed to examine the antibiotic prescribing rates for the three abovementioned outpatient conditions as the policy effects of the RDU Service Plan among hospitals that had different experiences with RDU activities. The present evaluation focused on a ministerial policy that has been implemented subsequent to the existing project with a similar aim of promoting rational antibiotic use.

\section{Ethics approval}

The study was approved by the Center for Ethics in Human Research, Khon Kaen University (HE601293).

\section{METHODS}

\section{Study design}

The levels of and trends in annual antibiotic prescribing rates were examined for fiscal years 2014-2016 (preService Plan) and fiscal years 2017-2019 (post-RDU Service

\section{RDU Service Plan implemented nationwide}

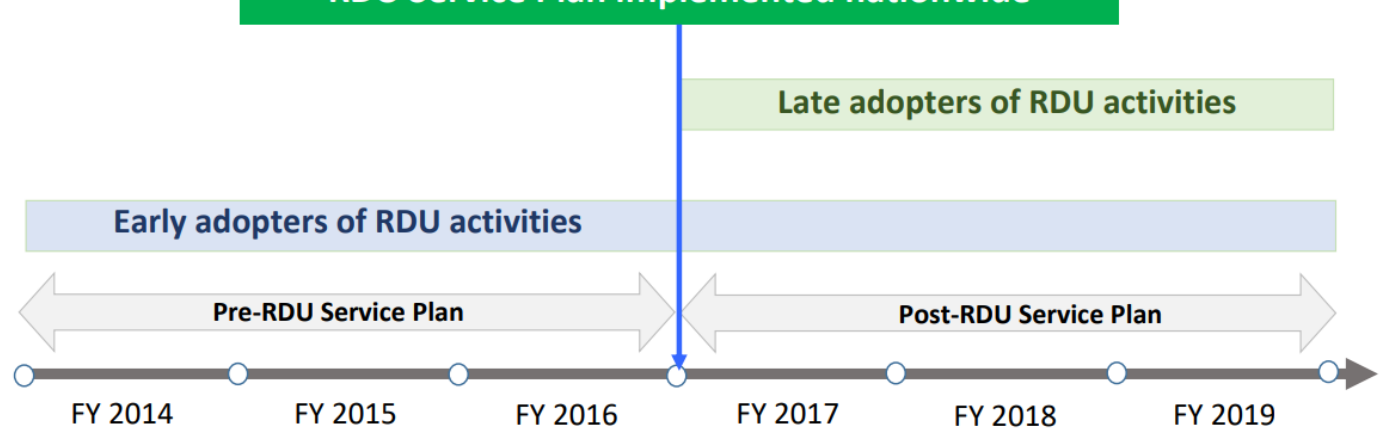

RDU Hospital Project

RDU Service Plan

RDU hospitals, $136 \mathrm{MOPH}$ hospitals Early adopters

\section{MOPH hospitals nationwide}

All remaining $\mathrm{MOPH}$ hospitals joined the RDU Service Plan and hospitals participating in the RDU Hospital Project continued their RDU activities through the RDU Service Plan.

Late adopters

Figure 1. Implementation of the rational drug use policies and analytical framework FY, fiscal year; MOPH, Ministry of Public Health; RDU, rational drug use 
Plan). As there are no data from before fiscal year 2014, the effects of the RDU Hospital Project as captured by pre- and post-policy changes in antibiotic prescribing levels and trends could not be estimated. As hospitals were distinguished between those participating in the RDU Hospital Project (namely, 'early adopters') and the remaining hospitals participating under the national RDU Service Plan (namely, 'late adopters') on the basis of the period of their involvement in RDU activities, the antibiotic prescribing levels and trends of these two hospital groups were compared. A quasi-experiment using a comparison of the pre-policy/post-policy changes in antibiotic prescribing levels and trends between the early adopter hospitals and the late adopter hospitals was used (Figure 1). Results from the comparisons of the pre-policy/post-policy levels and trends within each group were described.

\section{Data sources and measures}

In Thailand, a patient with a trivial infection may visit a hospital's outpatient department to obtain an antibiotic prescription that is usually filled by the pharmacy unit of the same hospital. In the present study, hospital-level data on the antibiotic prescribing rate as a percentage of outpatient visits for the three study conditions with diagnoses listed in the RDU handbook (Online appendix) were obtained from the hospitals that reported the data on an annual basis via the MOPH HDC. ${ }^{16}$ Notably, RIs included influenza, sinusitis, tonsillitis, pharyngitis, laryngitis, bronchitis, bronchiolitis, and otitis media but did not include pneumonia. To distinguish the study hospitals that were early adopters from the rest, exhaustive lists of individual hospitals participating in the RDU Hospital Project that were obtained from the FDA were linked to the antibiotic prescribing dataset from the MOPH HDC.

\section{Statistical analysis}

The antibiotic prescribing rates for each year were aggregated across individual hospitals using an equally weighted mean or, technically, an arithmetic mean, which was calculated as the summation of the prescribing rates across hospitals divided by the number of study hospitals. A mean was calculated for the group of early RDU activity adopters, and another was calculated for the rest of the hospitals belonging to the late adopter group. The dispersion in the antibiotic prescribing rates across varying numbers of hospitals per year was measured by the standard error (SE) of the group mean and was presented in terms of the $95 \%$ confidence interval (CI).

An interrupted time-series analysis was conducted, treating fiscal years 2014-2016 and 2017-2019 as the pre-policy and post-policy periods, respectively, with respect to the RDU Service Plan. The effect of the RDU Service Plan policy was captured by the immediate policy effect on changes in the levels of annual antibiotic prescribing rates in fiscal year 2017 and by additional changes in trends during the postpolicy periods (fiscal years 2017-2019), which were compared between the two hospital groups (early vs. late adopters) using a segmented linear regression. ${ }^{17}$ To account for serial correlation between the antibiotic

\begin{tabular}{|c|c|c|}
\hline & Early adopters $(\mathrm{n}=136)^{\mathrm{a}}$ & Late adopters $(\mathrm{n}=681)^{\mathrm{a}}$ \\
\hline \multicolumn{3}{|l|}{ Type of hospitals, number } \\
\hline District & 113 & 595 \\
\hline Provincial & 23 & 86 \\
\hline \multicolumn{3}{|l|}{ Geographic location of hospitals, number } \\
\hline Northern region & 46 & 137 \\
\hline Northeastern region & 62 & 234 \\
\hline Central region & 1 & 201 \\
\hline Southern region & 27 & 109 \\
\hline \multicolumn{3}{|c|}{ 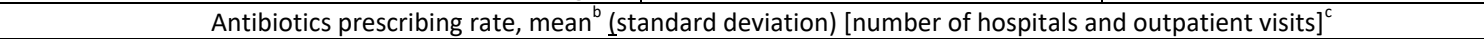 } \\
\hline \multicolumn{3}{|c|}{ Respiratory infections $^{\mathrm{d}} \mid$} \\
\hline Fiscal year 2014 & $41.5(14.6)[37,344564]$ & $43.5(15.4)[419,3087582]$ \\
\hline Fiscal year 2015 & $40.5(13.0)[78,693546]$ & $41.5(14.4)[478,3703112]$ \\
\hline Fiscal year 2016 & $36.7(12.2)[109,1088750]$ & $40.0(13.4)[607,5289537]$ \\
\hline Fiscal year 2017 & $31.3(11.0)[136,1219255]$ & $32.4(11.6)[677,5753129]$ \\
\hline Fiscal year 2018 & $24.4(8.4)[135,1170717]$ & $25.7(9.9)[679,5514038]$ \\
\hline Fiscal year 2019 & $21.0(6.7)[136,1086206]$ & $22.1(8.5)[681,5098334]$ \\
\hline \multicolumn{3}{|l|}{ Acute diarrhea } \\
\hline Fiscal year 2014 & $47.9(15.4)[37,65049]$ & $45.7(17.1)[419,624452]$ \\
\hline Fiscal year 2015 & $41.1(13.9)[78,130974]$ & 42.0 (16.7) $[477,794671]$ \\
\hline Fiscal year 2016 & $35.8(12.1)[109,207506]$ & 40.9 (15.9) [606, 1053024] \\
\hline Fiscal year 2017 & $29.7(10.9)[136,247117]$ & $32.2(13.6)[677,1163154]$ \\
\hline Fiscal year 2018 & $21.6(8.5)[135,259551]$ & $23.0(11.0)[678,1302709]$ \\
\hline Fiscal year 2019 & $17.9(7.0)[136,267546]$ & $19.3(9.0)[681,1251650]$ \\
\hline \multicolumn{3}{|l|}{ Fresh wounds } \\
\hline Fiscal year 2014 & $64.1(8.0)[37,122128]$ & 64.8 (9.7) [418, 1330707] \\
\hline Fiscal year 2015 & $62.0(8.7)[78,241451]$ & $65.1(9.8)[478,1710325]$ \\
\hline Fiscal year 2016 & $60.8(9.2)[109,402247]$ & $65.3(9.3)[606,2146376]$ \\
\hline Fiscal year 2017 & $57.3(9.8)[136,533471]$ & $62.0(9.7)[676,2633784]$ \\
\hline Fiscal year 2018 & $51.2(10.3)[135,569364]$ & $55.6(11.8)[677,2890100]$ \\
\hline Fiscal year 2019 & $41.3(11.7)[136,468507]$ & $45.6(12.5)[675,2506235]$ \\
\hline \multicolumn{3}{|c|}{$\begin{array}{l}{ }^{a} \text { Total number of hospitals overall study years } \\
{ }^{b} \text { Unweighted by the number of outpatient visits per hospital (or equally weighted) } \\
{ }^{c} \text { The number of hospitals submitting health service data to the Ministry of Public Health increased in later years } \\
\text { d Includes upper respiratory tract infections and acute bronchitis }\end{array}$} \\
\hline
\end{tabular}




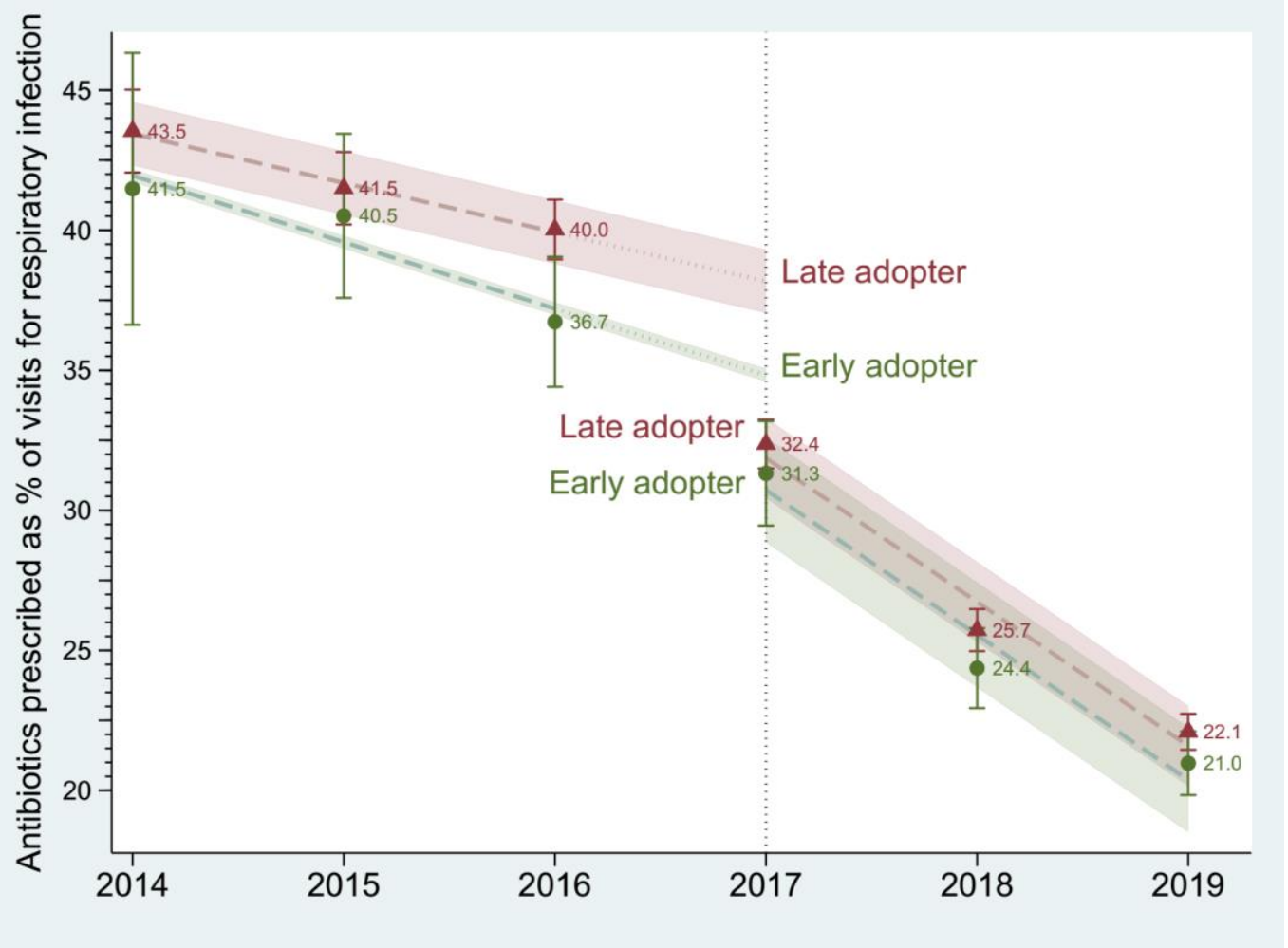

Figure 2. Observed levels of and predicted trends in antibiotic prescribing rates for respiratory infections, fiscal years 2014-2019 Round and triangle dots -Observed rates for early and late adopters, respectively, with the vertical, solid lines representing $95 \%$ confidence intervals for the mean rates (dots) across hospitals.

Slope, dashed lines: Predicted linear trends before and after the implementation of the RDU Service Plan policy (in fiscal year 2017; the vertical dotted line) for early and late adopters with shaded areas representing the $95 \%$ confidence interval for the point estimates of the predicted trends (dashed lines).

prescribing rates in adjacent years, a generalized least squares estimator that accounted for the first-order autoregression was employed using the Prais-Winsten transformation. ${ }^{18}$ Uncertainty in the predicted trends and in the point estimates of the policy effects was represented by $95 \% \mathrm{Cls}$. Statistical significance levels were determined for two-tailed, type-I error with an alpha of 0.05. All analyses were performed using Stata version 14 (StataCorp, College Station, Texas 77845 USA).

\section{RESULTS}

\section{Observed antibiotic prescribing rates}

Table 1 summarizes the number of study hospitals by type and geographic location and also summarizes the observed rates of antibiotic prescribing and the number of outpatient visits for each target condition during fiscal years 2014 to 2019.

In fiscal year 2014, the mean prescribing rate across hospitals was slightly lower than half of the outpatient visits for RIs and acute diarrhea (41.5 and 47.9\%, respectively) and was over half of the visits for fresh wounds (64.1\%) in the early adopter group. In the late adopter hospital group, the mean prescribing rates for RIs and for fresh wounds were slightly higher (43.5 and 64.8\%, respectively) than those in the early adopter group and the rate for acute diarrhea (45.7\%) was slightly lower. In fiscal year 2016, just one year prior to the implementation of the RDU Service Plan, the mean prescribing rates for RIs, acute diarrhea and fresh wounds decreased to $36.7,35.8$ and $60.8 \%$, respectively, among the early adopters, while those among the late adopters decreased to 40.0 and $40.9 \%$ for RIs and acute diarrhea, respectively, but increased slightly to $65.3 \%$ for fresh wounds. In fiscal year 2017, the first year of the RDU Service Plan implementation, there was a sharp drop in the antibiotic prescribing rates for all three conditions in both hospital groups (RIs, 31.3 and 32.4\%; acute diarrhea, 29.7 and $32.2 \%$; and fresh wounds, 57.3 and $62.0 \%$ for the early and late adopters, respectively). From the establishment of the RDU Service Plan onwards, the antibiotic prescribing rates for all three conditions in both the early and late adopter groups continued to decrease and reached their lowest rates in fiscal year 2019 (RIs, 21.0 and 22.1\%; acute diarrhea, 17.9 and 19.3\%; and fresh wounds, 41.3 and $45.6 \%$, respectively).

The standard deviations (SDs) shown alongside the mean values in Table 1 reflect the variability in antibiotic use across hospitals each year. Except for fresh wounds, variations in the observed prescribing rates across hospitals decreased gradually over time in both the early and late adopter groups.

\section{Trends in antibiotic prescribing pre- and post-RDU Service} Plan

Figure 2, Figure 3 and Figure 4 illustrate the predicted trends and their variation in the mean rates of antibiotic prescribing in the two hospital groups for RIs, acute diarrhea and fresh wounds pre- and post-RDU Service Plan. 


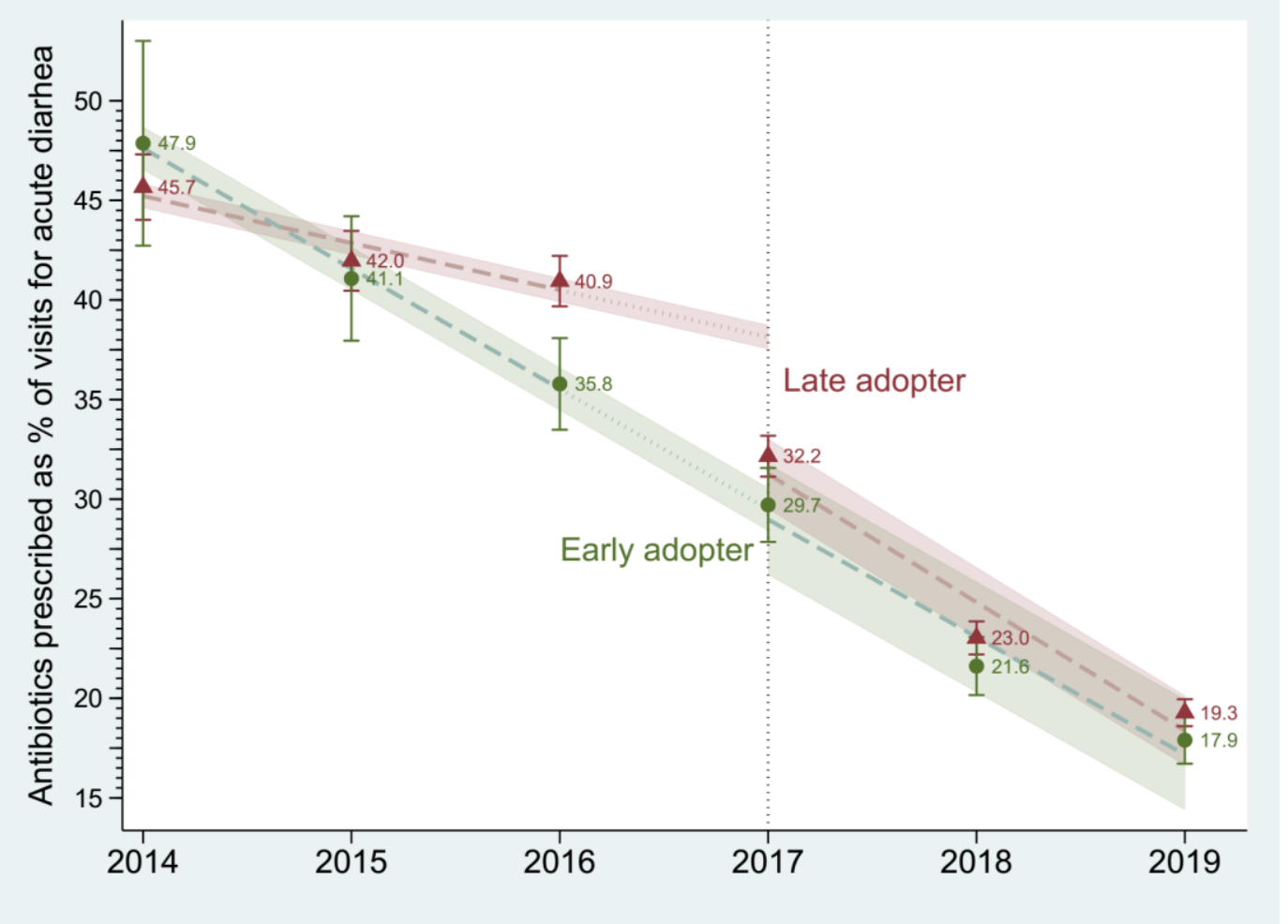

Figure 3. Observed levels of and predicted trends in antibiotic prescribing rates for acute diarrhea, fiscal years 2014-2019 Round and triangle dots: Observed rates for early and late adopters, respectively, with the vertical, solid lines representing $95 \%$ confidence intervals for the mean rates (dots) across hospitals.

Slope, dashed lines: Predicted linear trends before and after the implementation of the RDU Service Plan policy (in fiscal year 2017; the vertical dotted line) for early and late adopters with shaded areas representing the $95 \%$ confidence interval for the point estimates of the predicted trends (dashed lines).

For RIs and acute diarrhea, the $95 \% \mathrm{Cls}$ of the observed mean prescribing rates narrowed during the post-policy period as the standard errors (SEs) decreased and the number of study hospitals included each year increased. This was concordant with a decrease in variation in hospital antibiotic use, as shown previously by the SDs in Table 1 .

Antibiotic prescribing rates for RIs during the pre-RDU Service Plan period (fiscal years 2014-2016) decreased on average 2.4 and 1.8 percentage points per year $(p=0.015$ and <0.001) among the early and late adopters, respectively (Table 2 ). When the RDU Service Plan was introduced in fiscal year 2017, the prescribing levels in the two groups decreased abruptly by 4.1 and 6.3 percentage points ( $p=0.077$ and $<0.001)$. During the post-RDU Service Plan period (fiscal years 2017-2019), prescribing decreased at a similar rate of 5.2 and 5.1 percentage points per year $(p=0.002$ and 0.001$)$. Comparing the post-policy trends with the pre-policy trends, antibiotic prescribing for RIs decreased at statistically significant rates of 2.8 and 3.4 percentage points per year $(p=0.039$ and $<0.001)$ in the early and late adopter groups, respectively.

For acute diarrhea, the antibiotic prescribing rate during the pre-policy period decreased noticeably by 6.0 percentage points per year $(p<0.001)$ among the early adopters, whereas the rate among the late adopters decreased by 2.4 percentage points per year $(p<0.001)$. The magnitude of the decrease in the pre-policy trend among the early adopters was larger than that among the late adopters by 3.7 percentage points $(p=0.004)$. In the first year of the RDU Service Plan, antibiotic prescribing among the late adopters decreased rapidly by 6.9 percentage points $(p=0.001)$, whereas that among the early adopters decreased by only 0.51 percentage points $(p=0.754)$. During the post-policy period, the prescribing rates in the late and early adopter groups continued to decrease (6.4 and 5.9 percentage points per year, $p=0.004$ and 0.003 , respectively), resulting in changes relative to the pre-policy trends of -4.1 and +0.14 percentage points $(p<0.001$ and $=0.891)$, respectively.

For fresh wounds, the pre-policy trends between the two hospital groups differed by 1.9 percentage points $(p=0.001)$, as the antibiotic prescribing rate among the early adopters decreased by 1.6 percentage points per year $(p=0.001)$ but that among the late adopters increased by 0.28 percentage points per year $(p<0.001)$. In the first year of the RDU Service Plan, the prescribing rate among the early adopters decreased slightly by 1.1 percentage points $(p=0.442)$, while that among the late adopters decreased abruptly by 3.0 percentage points $(p=0.007)$. The postpolicy trend showed a similar and rapid decrease in antibiotic prescribing between the two groups ( 8.0 vs. 8.2 percentage points per year) and a further reduction relative to the pre-policy trends of 6.4 and 8.5 percentage points ( $p=0.001$ and $<0.001$ ).

\section{Effects of the RDU Service Plan}

For the late adopters, an immediate policy effect during the first year of the RDU Service Plan implementation (fiscal year 2017) was noticeable in the significantly abrupt drop 


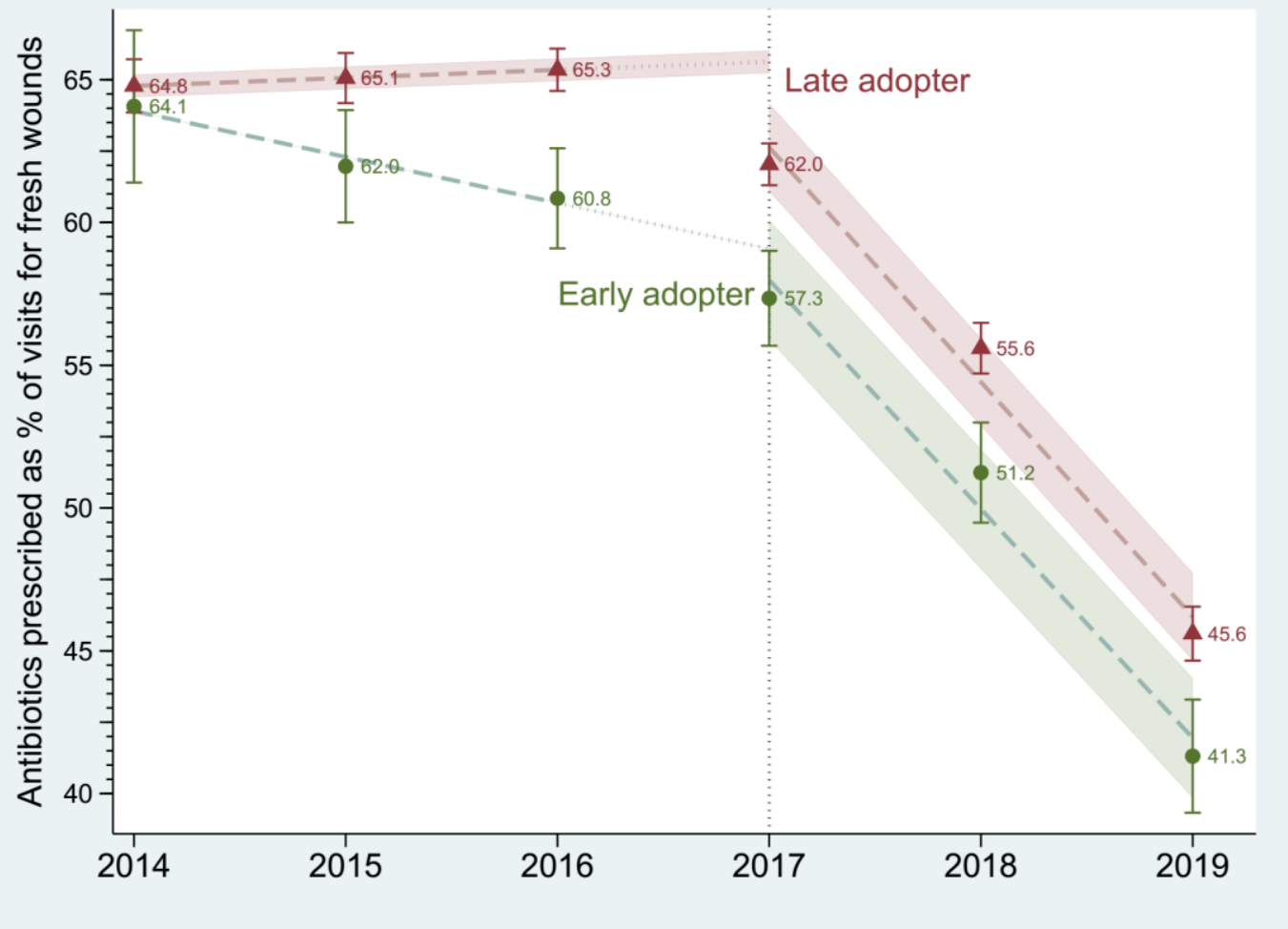

Figure 4. Observed levels of and predicted trends in antibiotic prescribing rates for fresh wounds, fiscal years 2014-2019 Round and triangle dots: Observed rates for early and late adopters, respectively, with the vertical, solid lines representing 95\% confidence intervals for the mean rates (dots) across hospitals.

Slope, dashed lines: Predicted linear trends before and after the implementation of the RDU Service Plan policy (in fiscal year 2017; the vertical dotted line) for early and late adopters with shaded areas representing the $95 \%$ confidence interval for the point estimates of the predicted trends (dashed lines).

in the antibiotic prescribing level for all three conditions. A medium-term effect of the RDU Service Plan policy was shown in the greater magnitude of the continually decreasing trend in fiscal years 2017-2019 than that of the decreasing trend in fiscal years 2014-2016 for all three conditions in both study groups, except for acute diarrhea in the early adopter group.

Comparing antibiotic prescriptions for RIs between the two hospital groups, the level of and trend in antibiotic prescribing decreased more rapidly among late adopters than among early adopters after the RDU Service Plan was established, by 2.2 percentage points for the difference in the level changes in fiscal year 2017 and by 0.58 percentage points for the difference in the trend changes in fiscal years 2017-2019. Differences in the magnitude of the policy effects between the two groups, however, were not very large and did not reach statistical significance $(p=0.329$ and 0.630 , respectively).

For acute diarrhea, the drop in the level of prescribing rates in fiscal year 2017 among the late adopters was noticeably greater in magnitude than that among the early adopters, a difference of 6.4 percentage points, which is a marginally significant immediate policy effect $(p=0.071)$. The betweengroup difference in the decrease in the prescribing trend

\begin{tabular}{|c|c|c|c|c|c|}
\hline \multirow[b]{2}{*}{$\%$ points $(95 \% \mathrm{Cl})$} & \multicolumn{2}{|c|}{ Pre-policy (fiscal years 2014-2016) } & \multicolumn{3}{|c|}{ Post-policy (fiscal years $2017-2019$ ) } \\
\hline & Baseline rate $^{t}$ & Trend (c) & $\begin{array}{l}\text { Change in levels } \\
\text { (fiscal year 2017) }\end{array}$ & Trend (d) & Change in trends $(d-c)$ \\
\hline \multicolumn{6}{|l|}{ Respiratory infections } \\
\hline Late adopters (a) & $45.2^{* *}(44.7,45.7)$ & $-1.8^{* *}(-1.5,-2.0)$ & $-6.3 * *(-4.4,-8.2)$ & $-5.1 * *(-3.4,-6.8)$ & $-3.4 * *(-2.2,-4.6)$ \\
\hline Early adopters (b) & $44.3^{* *}(40.6,48.0)$ & $-2.4 *(-0.78,-4.0)$ & $-4.1(-8.9,0.71)$ & $-5.2 * *(-3.2,-7.2)$ & $-2.8 *(-0.23,-5.4)$ \\
\hline Difference $(a-b)$ & $0.88(-2.9,4.6)$ & $0.62(-1.0,2.2)$ & $-2.2(-7.7,3.3)$ & $0.036(-2.6,2.7)$ & $-0.58(-3.7,2.5)$ \\
\hline \multicolumn{6}{|l|}{ Acute diarrhea } \\
\hline Late adopters (a) & $47.6^{* *}(45.1,50.1)$ & $-2.4^{* *}(-1.3,-3.4)$ & $-6.9 * *(-2.8,-11.0)$ & $-6.4 * *(-3.4,-9.5)$ & $-4.1 * *(-1.7,-6.5)$ \\
\hline Early adopters (b) & $53.7^{* *}(51.7,55.6)$ & $-6.0 * *(-5.2,-6.9)$ & $-0.51(-4.8,3.8)$ & $-5.9 * *(-3.4,-8.4)$ & $0.14(-2.5,2.8)$ \\
\hline Difference $(a-b)$ & $-6.1 *(-10.1,2.1)$ & $3.7^{* *}(1.9,5.4)$ & $-6.4(-13.6,0.85)$ & $-0.54(-4.5,3.4)$ & $-4.2(-8.5,0.077)$ \\
\hline \multicolumn{6}{|l|}{ Fresh wounds } \\
\hline Late adopters (a) & $64.50 * *(64.48,64.51)$ & $0.28 * *(0.27,0.29)$ & $-3.0 * *(-0.82,-5.2)$ & $-8.2 * *(-6.2,-10.2)$ & $-8.5 * *(-7.1,-9.9)$ \\
\hline Early adopters (b) & $65.5^{* *}(64.2,66.8)$ & $-1.6^{* *}(-1.1,-2.2)$ & $-1.1(-4.6,2.5)$ & $-8.0 * *(-5.8,-10.2)$ & $-6.4 * *(-4.2,-8.6)$ \\
\hline Difference $(a-b)$ & $-1.01(-2.3,0.27)$ & $1.9 * *(1.3,2.4)$ & $-1.9(-6.6,2.8)$ & $-0.20(-3.2,2.8)$ & $-2.1(-5.1,0.92)$ \\
\hline
\end{tabular}


post-policy was equal to 4.2 percentage points, which was borderline significant ( $p=0.053)$.

For fresh wounds, the policy effect captured by the between-group difference in the immediate decrease in antibiotic prescribing levels in fiscal year 2017 was 1.9 percentage points $(p=0.323)$. The magnitude of the decrease in the prescribing trend post-policy among the early adopters was smaller than that among the late adopters by 2.1 percentage points, a statistically nonsignificant policy effect $(p=0.126)$.

\section{DISCUSSION}

For the late adopters, the RDU Service Plan was able to substantially decrease both the level of and trend in antibiotic prescribing rates for all three conditions; thus, the RDU Service Plan augmented the preexisting trends. The effect of the RDU Service Plan beyond that of the RDU Hospital Project among the early adopters was noticeable for Rls and fresh wounds, for which post-policy trends in antibiotic prescribing decreased further and with a significantly higher magnitude than that of the decrease in pre-policy trends. The magnitude of the policy effect of the RDU Service Plan among the late adopters was greater than that among the early adopters for all three conditions, even though the differences were not statistically significant. The RDU Service Plan was able to reduce antibiotic prescribing for RIs and acute diarrhea close to the $20 \%$ goal and that for fresh wounds close to the $40 \%$ goal within three years in both groups.

Apart from a decreasing trend in mean values, the variation in prescribing rates across hospitals narrowed. A gradual decrease in the SDs and SEs for RIs and acute diarrhea implies improved uniformity in prescribing practices across hospitals in both the early and late adopter groups after RDU policy implementation.

During the RDU Hospital Project campaign, antibiotic prescribing for RIs and acute diarrhea decreased in both the early and late adopter groups and at a more rapid rate among the early adopters. A relatively smaller decrease in the trends in antibiotic prescribing in the late adopter group for RIs and acute diarrhea might be due to a mix between spillovers from the RDU activities of the early adopters and the pre-existing decreasing trend within the late adopter group. This implies that the hospitals in both groups were aware of antibiotic use problems and were ready to bring about changes.

Regarding fresh wounds, the policy effect might be confounded by the possibility of prescribing antibiotics for contaminated wounds, which the International Statistical Classification of Diseases and Related Health Problems (ICD), $10^{\text {th }}$ Revision codes do not distinguish from clean wounds.

The results of our study conform with those of previous studies conducted in China, Slovenia, and France, which assessed nationwide, multifaceted policy interventions. ${ }^{19-21}$ These studies reported a decrease in the quantity of antibiotics used in the general population while our study focused its assessment on patients. It has been reported that policy impacts increase as selected policies are implemented at the national level. ${ }^{19-23}$ Our study did not disentangle the effects of specific interventions, as the RDU Service Plan contained numerous interventions and was implemented on different levels, for example, awareness campaigns, RDU handbooks, and hospital strategies to conform to RDU criteria. Therefore, recommendations based on the comparative effectiveness of each activity within this multifaceted policy cannot be provided for other countries.

Rigorous evidence on the impact of antibiotic use policies at the national level was limited. The available reports are mainly descriptive, and most study designs consist of a onegroup posttest only or a pre-policy and post-policy comparison without controls. ${ }^{19,24}$ In our study, the first hospital group that participated in the RDU Hospital Project served as the comparison group, and there were comparisons within each group before the policy, during implementation and over the medium term. However, there were no hospitals that did not adopt the RDU policies (i.e., there was no null-control group) throughout the study period. It is important to note that the antibiotic prescribing rates after policy implementation were measured for three years, which is a medium-term duration. However, statistically significant policy effects were apparent. Potential confounding factors such as hospital types and geographic locations were not accounted for in the analysis. Although the number of hospitals varied across time points, the $95 \% \mathrm{Cls}$ for the antibiotic prescribing rates hinted that the variation across hospitals was modest even during the pre-policy years when there were fewer participating hospitals. Another limitation is the inability to detect if there was seasonal variation in antibiotic prescribing since the hospital-level data used for the analysis were based on yearly rates.

Regarding long-term effects, because prescribing rates continued to decrease at similar rates between the late and early adopters, a long-term effect can be expected. Early adopters who implemented the RDU activities beginning in 2014 continued to experience a decline in their prescribing rates at the end of the study (2019). Further studies should evaluate the long-term effects of this ministerial policy. To gain a better understanding of the root causes, in-depth analyses are warranted, and prescribing rates specific to subgroups of the diagnosed conditions of RIs should be further examined.

As antibiotics available in community pharmacies in Thailand do not require prescriptions, overutilization due to self-medication is also an issue. This problem has been found in several countries. ${ }^{25-27}$ Mandatory policies on the prohibition of antibiotic sales without prescriptions have been effective. ${ }^{25}$ Previous studies have reported that programs using multifaceted interventions, including training and educational materials, to promote the rational use of antibiotics in community pharmacies are also effective. ${ }^{28-29}$ Further policy interventions in the community pharmacy context are warranted.

\section{CONCLUSIONS}

In conclusion, the RDU Service Plan was an effective policy for decreasing antibiotic prescribing for questionable 
Waleekhachonloet O, Rattanachotphanit T, Limwattananon C, Thammatacharee N, Limwattananon S. Effects of a national policy advocating rational drug use on decreases in outpatient antibiotic prescribing rates in Thailand. Pharmacy Practice 2021 JanMar;19(1):2201.

https://doi.org/10.18549/PharmPract.2021.1.2201

conditions common in outpatient visits. This nationwide ministerial intervention also exhibited an add-on policy effect to the RDU Hospital Project, in which selected hospitals participated voluntarily.

\section{CONFLICT OF INTEREST}

All authors declare that they have no conflict of interest.

\section{FUNDING}

This study was funded by Health Systems Research Institute, Thailand.

\author{
AUTHOR ROLES (CRediT) \\ Conceptualization: OW, CL, NT. \\ Formal analysis: $\mathrm{OW}, \mathrm{CL}$. \\ Funding acquisition: $\mathrm{CL}$. \\ Methodology: OW, TR, CL. \\ Supervision: SL. \\ Validation: SL. \\ Writing - original draft: $\mathrm{OW}, \mathrm{TR}, \mathrm{CL}$ \\ Writing - review \& editing: CL.
}

References

1. Klein EY, Van Boeckel TP, Martinez EM, et al. Global increase and geographic convergence in antibiotic consumption between 2000 and 2015. Proc Natl Acad Sci U S A. 2018;115(15):E3463-E3470. https://doi.org/10.1073/pnas.1717295115

2. Sumpradit N, Suttajit S, Poonplosup S, Chuancheun R, Prakongsai P. Landscape of antimicrobial resistance situations and actions in Thailand by Bureau of Drug Control. http://www.fda.moph.go.th/sites/drug/Shared\%20Documents/AMR/06.pdf (accessed Jun 15, 2018).

3. Suda KJ, Hicks LA, Roberts RM, Hunkler RJ, Matusiak LM, Schumock GT. Antibiotic Expenditures by Medication, Class, and Healthcare Setting in the United States, 2010-2015. Clin Infect Dis. 2018;66(2):185-190. https://doi.org/10.1093/cid/cix773

4. Mikulic M. U.S.Total medicine spending 2002 -2019. https://www.statista.com/statistics/238689/us-total-expenditure-onmedicine/ (accessed Dec 25, 2020).

5. Chanvatik S, Kosiyaporn H, Lekagul A, et al. Knowledge and use of antibiotics in Thailand: A 2017 national household survey. PLoS One. 2019;14(8):e0220990. https://doi.org/10.1371/journal.pone.0220990

6. Sumpradit N, Chongtrakul P, Anuwong K, et al. Antibiotics Smart Use: a workable model for promoting the rational use of medicines in Thailand. Bull World Health Organ. 2012;90(12):905-913. https://doi.org/10.2471/blt.12.105445

7. World Health Organization. The Pursuit of Responsible Use of Medicines: Sharing and Learning from Country Experiences. http://apps.who.int/iris/bitstream/10665/75828/1/ (accessed Jun 15, 2018).

8. Rational Drug Use Sub-Committee. Rational drug use hospital manual. Nonthaburi: The Agricultural Cooperative Federation of Thailand; 2015

9. Chongtrakul P. RDU hospital: the pathway to rational drug use. Thai J Pharmacol. 2015; 37(1):48-62

10. Shayakul C, Leela-Udomlipi S, Wannakul W, Bunupuradah P, Tanta N, Putchakarn P, Puripunyawanich N, Wananukul W, Tandayya N, Thammawut W. Development of rational drug use hospital. https://kb.hsri.or.th/dspace/handle/11228/4902?locale-attribute=th (accessed Jun 15, 2018).

11. Health Administration Division. Service Plan: Rational drug use manual. http://dmsic.moph.go.th/dmsic/admin/files/userfiles/files/Manual Service\%20Plan\%20RDU Sept2016.pdf (accessed May 1, 2017).

12. World Health Organization. How to investigate drug use in health facilities: selected drug use indicators. https://apps.who.int/medicinedocs/en/d/Js2289e/ (accessed Jun 15, 2018).

13. Adriaenssens N, Coenen S, Tonkin-Crine S, et al. European Surveillance of Antimicrobial Consumption (ESAC): diseasespecific quality indicators for outpatient antibiotic prescribing. BMJ Qual Saf. 2011;20(9):764-772. https://doi.org/10.1136/bmjas.2010.049049

14. International Wound Infection Institute. Wound Infection in Clinical Practice. https://www.woundsinternational.com/resources/details/iwii-wound-infection-clinical-practice (accessed Jun 15, 2018).

15. Sirijatuphat $R$, Choochan T, Siritongtaworn $P$, Sripojtham V, Thamlikitkul V. Implementation of antibiotic use guidelines for fresh traumatic wound at Siriraj Hospital. J Med Assoc Thai. 2015;98(3):245-252

16. Health Data Center. Ministry of Public Health. RDU Service Plan indicators. https://hdcservice.moph.go.th/hdc/reports/page.php?cat id=03b912ab9ccb4c07280a89bf05e5900e (accessed Dec 1 , 2019)

17. Bernal JL, Cummins S, Gasparrini A. Interrupted time series regression for the evaluation of public health interventions: a tutorial. Int J Epidemiol. 2017;46(1):348-355. https://doi.org/10.1093/ije/dyw098

18. Prais SJ, Winsten CB. Trend estimators and serial correlation. http://cowles.econ.yale.edu/P/ccdp/st/s-0383.pdf (accessed Jun 15, 2018).

19. Xiao Y, Zhang J, Zheng B, Zhao L, Li S, Li L. Changes in Chinese policies to promote the rational use of antibiotics. PLoS Med. 2013;10(11):e1001556. https://doi.org/10.1371/journal.pmed.1001556

20. Fürst J, Čižman M, Mrak J, et al. The influence of a sustained multifaceted approach to improve antibiotic prescribing in Slovenia during the past decade: findings and implications. Expert Rev Anti Infect Ther. 2015;13(2):279-289. https://doi.org/10.1586/14787210.2015.990381 
Waleekhachonloet O, Rattanachotphanit T, Limwattananon C, Thammatacharee N, Limwattananon S. Effects of a national policy advocating rational drug use on decreases in outpatient antibiotic prescribing rates in Thailand. Pharmacy Practice 2021 JanMar;19(1):2201.

https://doi.org/10.18549/PharmPract.2021.1.2201

21. Bernier A, Delarocque-Astagneau E, Ligier C, Vibet MA, Guillemot D, Watier L. Outpatient antibiotic use in France between 2000 and 2010: after the nationwide campaign, it is time to focus on the elderly. Antimicrob Agents Chemother. 2014;58(1):71-77. https://doi.org/10.1128/aac.01813-13

22. Holloway KA, Rosella L, Henry D. The Impact of WHO Essential Medicines Policies on Inappropriate Use of Antibiotics. PLoS One. 2016;11(3):e0152020. https://doi.org/10.1371/journal.pone.0152020

23. Holloway KA, Henry D. WHO essential medicines policies and use in developing and transitional countries: an analysis of reported policy implementation and medicines use surveys. PLoS Med. 2014;11(9):e1001724. https://doi.org/10.1371/journal.pmed.1001724

24. Ho M, Hsiung CA, Yu HT, Chi CL, Chang HJ. Changes before and after a policy to restrict antimicrobial usage in upper respiratory infections in Taiwan. Int J Antimicrob Agents. 2004;23(5):438-445. https://doi.org/10.1016/.ijantimicag.2003.10.013

25. Santa-Ana-Tellez Y, Mantel-Teeuwisse AK, Dreser A, Leufkens HG, Wirtz VJ. Impact of over-the-counter restrictions on antibiotic consumption in Brazil and Mexico. PLoS One. 2013;8(10):e75550. https://doi.org/10.1371/journal.pone.0075550

26. Zajmi D, Berisha M, Begolli I, et al. Public knowledge, attitudes and practices regarding antibiotic use in Kosovo. Pharm Pract (Granada). 2017;15(1):827. https://doi.org/10.18549/pharmpract.2017.01.827

27. Torres NF, Solomon VP, Middleton LE. Pharmacists' practices for non-prescribed antibiotic dispensing in Mozambique. Pharm Pract (Granada). 2020;18(3):1965. https://doi.org/10.18549/pharmpract.2020.3.1965

28. Gastelurrutia MA, Larrañaga B, Ortega B. First program on rational use of antibiotics in Gipuzkoa. Assessment of 19992004 period. Pharm Pract (Granada). 2006;4(1):1-8.

29. Gastelurrutia MA, Larrañaga B, Garay A, Echeveste Fde A, Fernandez-Llimos F. Impact of a program to reduce the dispensing of antibiotics without a prescription in Spain. Pharm Pract (Granada). 2013;11(4):185-190. https://doi.org/10.4321/s1886-36552013000400002 